\title{
Oxaliplatin use in pressurized intraperitoneal aerosol chemotherapy (PIPAC) is safe and effective: A multicenter study
}

\author{
Olivia Sgarbura ${ }^{\text {a, b, }}{ }^{*}$, Martin Hübner ${ }^{c}$, Mohammad Alyami ${ }^{\text {d, e }}$, Clarisse Eveno ${ }^{\text {, }}$ \\ Johan Gagnière ${ }^{\mathrm{g}}$, Basile Pache ${ }^{\mathrm{c}}$, Marc Pocard ${ }^{\mathrm{h}}$, Naoual Bakrin ${ }^{\mathrm{d}}$, François Quénet ${ }^{\mathrm{a}, \mathrm{b}}$ \\ a Department of Surgical Oncology, Cancer Institute Montpellier (ICM), Montpellier, France \\ b University of Montpellier, France \\ ${ }^{\mathrm{c}}$ Department of Visceral Surgery, Lausanne University Hospital (CHUV), Lausanne, Switzerland \\ d Department of General Surgery and Surgical Oncology, Lyon Sud University Hospital, Pierre Benite, France \\ e Department of General Surgery and Surgical Oncology, King Khalid Hospital, Najran, Saudi Arabia \\ ${ }^{\mathrm{f}}$ Department of General Surgery, University Hospital Lille, Lille, France \\ ${ }^{g}$ Centre Hospitalier Universitaire Clermont-Ferrand, France \\ h INSERM U1275, CAP Paris-Tech, Carcinomatosis Peritoneum Paris Technology, Lariboisière Hospital, AP-HP, Paris 7- Diderot University, Sorbonne Paris \\ Cité, France
}

\section{A R T I C L E I N F O}

\section{Article history:}

Received 6 March 2019

Received in revised form

26 April 2019

Accepted 8 May 2019

Available online 9 May 2019

\section{Keywords:}

PIPAC

Oxaliplatin

Peritoneal cancer

Intraperitoneal chemotherapy

Tolerance

\begin{abstract}
A B S T R A C T
Introduction: Pressurized intraperitoneal aerosol chemotherapy (PIPAC) is a new drug delivery method used in patients with peritoneal cancer (PC) of primary or secondary origin. Intraperitoneal use of oxaliplatin raises concerns about toxicity, especially abdominal pain. The objective of this study was to assess the tolerance of PIPAC with oxaliplatin (PIPAC-Ox) in a large cohort of patients and to identify the risk factors for high grade toxicity, discontinuation of treatment and impaired survival.

Material and methods: This retrospective cohort study included all consecutive patients treated with PIPAC-Ox $\left(92 \mathrm{mg} / \mathrm{m}^{2}\right)$ in five centers specialized in the treatment of PC. The procedure was repeated every 6 weeks. Outcomes of interest were Common Terminology Criteria for Adverse Events (CTCAE), symptoms and survival (Kaplan-Meier). Univariate risk factors were included in a multinominal regression model to control for bias.

Results: Overall, 251 PIPAC-Ox treatments were performed in 101 patients (45 female) having unresectable PC of various origins: 66 colorectal, 15 gastric, 5 ovarian, 3 mesothelioma, 2 pseudomyxoma, 10 other malignancies (biliary, pancreatic, endocrine) respectively. The median PCI was 19 (IQR: 10-28). Postoperative abdominal pain was present in 23 patients. Out of the 9 patients with grade 3 abdominal pain, only 3 needed a change of PIPAC drug. CTCAE 4.0 toxicity grade 4 or higher was encountered in $16(15.9 \%)$ patients. The patients had a mean of 2.5 procedures/patient $(S D=1.5) .50$ subjects presented with symptom improvement.

Conclusions: Oxaliplatin-based PIPAC appears to be a safe treatment that offers good symptom control and promising survival for patients with advanced peritoneal disease.

(C) 2019 Elsevier Ltd, BASO The Association for Cancer Surgery, and the European Society of Surgical
\end{abstract} Oncology. All rights reserved.

\section{Introduction}

Pressurized intraperitoneal aerosol chemotherapy (PIPAC) is a new mode of minimal-invasive intraperitoneal (IP) drug delivery

\footnotetext{
* Corresponding author. Cancer Institute Montpellier, Surgical Oncology Department, 208, Avenue des Apothicaires, 34298, Cedex 01, Montpellier, France. E-mail address: olivia.sgarbura@icm.unicancer.fr (O. Sgarbura).
}

for patients with peritoneal cancer (PC) [1]. The current recommendations foresee at least 3 PIPAC treatments (4-6 weeks interval) as single modality or in combination with systemic chemotherapy(2).

Two regimens of IP agents are usually delivered through PIPAC: cisplatin in combination with doxorubicin (C/D) or oxaliplatin as monotherapy. A potential third agent is mitomycine $C$ but reports are scarce for this drug(2). For any of these agents, initial doses were empirically defined in the lower range in order to avoid 


$\begin{array}{ll}\text { List of abbreviations } \\ \text { ASA } & \text { American Society of Anesthesiology } \\ \text { AUC } & \text { area under the curve } \\ \text { BMI } & \text { body mass index } \\ \text { CRS } & \text { cytoreductive surgery } \\ \text { CTCAE } & \text { common terminology criteria for adverse events } \\ \text { HIPEC } & \text { heated intraperitoneal chemotherapy } \\ \text { IQR } & \text { interquartile range } \\ \text { IP } & \text { intraperitoneal } \\ \text { MPM } & \text { malignant peritoneal mesothelioma } \\ \text { OS } & \text { overall survival } \\ \text { PCI } & \text { peritoneal cancer index } \\ \text { PIPAC } & \text { pressurized intraperitoneal aerosol chemotherapy } \\ \text { PC } & \text { peritoneal cancer } \\ \text { PM } & \text { peritoneal metastases } \\ \text { PMP } & \text { pseudomyxoma peritonei }\end{array}$

systemic toxicities(3). Recently, a dose-finding study allowed to safely increase C/D doses by $40 \%$ (C: $10.5 \mathrm{mg} / \mathrm{m} 2$, D: $2.1 \mathrm{mg} / \mathrm{m} 2$ ) [4]. Currently, two phase I clinical trials are ongoing aiming to define the optimal dose for oxaliplatin (currently: $92 \mathrm{mg} / \mathrm{m} 2$ or $20 \%$ of the Elias regimen(5)) [6,7].

PIPAC was evaluated independently by different groups confirming consistently feasibility, safety, and good tolerance of the procedure(8-10). However, PIPAC-Ox was reluctantly used for fear of toxicity (especially abdominal pain) which was encountered after IP administration of oxaliplatin by means of an intraperitoneal catheter(11) or in form of heated intraperitoneal chemotherapy (HIPEC) [12]. So far, the largest experience on PIPAC-Ox included 17 patients with PM of colorectal origin having 48 PIPAC-Ox administrations (mean 2.8). CTCAE grade 3 toxicity was observed in $23 \%$ of patients but no CTCAE grade 4 was reported(13). In the French cohort analyzed at the end of the 1 st year of use of PIPAC, CTCAE 4.0 grade 3 toxicities were only found in $9,7 \%$ of patients having received either cisplatin-doxorubicin, oxaliplatin or mitomycine $\mathrm{C}$. While these initial studies were encouraging, they still convey limited information concerning the use of PIPAC-Ox regarding safety, tolerance and efficacy.

The aim of the present multicenter study was to assess the tolerance profile of PIPAC-Ox in a large cohort of patients and to identify risk factors for high grade toxicity, discontinuation of treatment and poor survival.

\section{Material and methods}

\section{Study design}

This is a multicenter retrospective cohort including all consecutive patients treated with PIPAC-Ox in five investigating centers (Montpellier Cancer Institute, Lausanne University Hospital, Lyon Sud University Hospital, Clermont Ferrand University Hospital, Lariboisière University Hospital) from January 2015 to December 2017. The study was approved by the Ethics Committee of the collecting center in accordance with the ethical standards of the Helsinki Declaration of 1975. Data was retrieved from prospectively maintained institutional data bases with permission of the respective institutional review boards and analyzed anonymously. Follow-up for survival data was performed until March 2018.

\section{PIPAC procedure}

All centers followed the structured PIPAC training program before the start of the clinical program and four of them became PIPAC training centers. The procedures were performed under general anesthesia and the standard protocol was described in detail elsewhere(14). Briefly, a first trocar was placed using open technique. Two trocars were used in total: one for the endoscope and one for the aerosolizer (Capnopen ${ }^{\odot}$; Capnomed GmbH, Villigendorf, Germany). The latter was connected to a high-pressure injector that applied up to 20 bar and a flow of $0.5 \mathrm{ml} / \mathrm{s}$. A pressurized aerosol containing oxaliplatin at a dose of $92 \mathrm{mg} / \mathrm{m} 2$ body surface in a $150-\mathrm{ml} 5 \%$ dextrose solution was applied via the injector and the aerosolizer. After the injection, therapeutical capnoperitoneum was maintained for $30 \mathrm{~min}$ at $37^{\circ} \mathrm{C}$. The remaining toxic aerosol was exsufflated in a closed aerosol wasting system(15). The procedure was repeated at 6 week intervals. Patients were managed postoperatively in the surgical unit. A visual analogue scale (VAS) was used for assessment of the pain. In the immediate postoperative setting the assessment was performed four times daily. The postoperative assessment was performed at the 3 week evaluation by the surgeon in charge. All the measures were translated in the CTCAE grading system by the patient's surgeon.

\section{Outcome measures}

Demographic, clinical, disease-related and treatment-related variables were collected for all patients. Symptomatic response to treatment, evolution of the peritoneal carcinomatosis index (PCI), reasons for discontinuation of the treatment were taken into account for each PIPAC. Adverse events were graded according to CTCAE version 4.0. Survival was calculated in months reported to the date of diagnosis of the primary disease, of the peritoneal carcinomatosis and of the first PIPAC treatment. Minimal follow-up was 3 months.

\section{Statistical analysis}

Continuous and categorical variables were collected and analyzed. Continuous data was reported as a median with an interquartile range except for the number of PIPAC procedures where the mean and the standard deviation were used for comparative purposes with previous reports. Frequencies were reported as raw numbers and percentages of the entire population of patients. Non parametric tests were used for comparison of independent variables (Mann-Whitney test, Chi-square). Regression analyses was used to test potential relationship between variables and one way ANOVA as well as multivariate analysis were performed for identifying potential prognostic factors. Regression analysis investigated the role of gender, elder age, $\mathrm{BMI}<20$, primary tumor in the onset of severe abdominal pain (>grade 2 ) or on CTCAE complication grade 3 or higher. Univariate analysis aimed to determine if any of the following factors were potentially prognostic for the early discontinuation of the treatment (defined as the administration of $<3$ PIPAC) or for the impaired survival: gender, $\mathrm{BMI}<20$, primary tumor, presence of abdominal pain CTCAE grade 1 to 3 , presence of any grade 3 toxicities. Survival analysis was performed by use of the Kaplan Meier model. A p-value $<0.05$ was considered significant. All data were analyzed with the SPSS software (SPSS 17.0; SPSS Inc., Chicago, Illinois, USA) for Windows.

\section{Results}

Between January 2015 and December 2017, 101 patients were 
Table 1

Demographic and clinical characteristics of the patients.

\begin{tabular}{lll}
\hline Variable & Subtype & Value (\%) \\
\hline Gender & Male & $56(55.4 \%)$ \\
Age & Female & $45(44.6 \%)$ \\
ASA & Median (IQR) & $59(50-70 / 5)$ \\
& I & $13(12.9 \%)$ \\
& II & $58(57.4 \%)$ \\
BMI & III & $21(20.8 \%)$ \\
Histology & Missing & $9(8.9 \%)$ \\
& Median (IQR) & $23(20.5-25.7)$ \\
& $<20$ & $22(21.8 \%)$ \\
Colorectal cancer & $66(65.4 \%)$ \\
Synchronicity & Gastric cancer & $15(14.8 \%)$ \\
& Ovarian cancer & $5(4.9 \%)$ \\
Previous chemotherapy & Peritoneal mesothelioma (MPM) & $3(3 \%)$ \\
& Peritoneal pseudomyxoma (PMP) & $2(2 \%)$ \\
Symptoms related to PM & Other ${ }^{*}$ & $10(9,9 \%)$ \\
& Median (IQR) & $19(10-28)$ \\
& Synchronous & $46(45,5 \%)$ \\
& Mescites & $53(52,5 \%)$ \\
& Pain & $2(2 \%)$ \\
& Impaired bowel function & $93(92,1 \%)$ \\
& Missing & $8(7,9 \%)$ \\
& No & $46(45,5 \%)$ \\
& Pachous & $23(22,8 \%)$ \\
\hline
\end{tabular}

*other histologies represent rare indications of PIPAC such as small bowel cancer, pancreatic cancer, cholangiocarcinoma, mixed neuroendocrine and carcinoma).

treated with 251 PIPAC-Ox in total. Demographics and clinical details are displayed in Table 1. All patients had histologically proven peritoneal metastases with a median PCI of 19 (IQR:10-28) at the start of treatment.

Patients had a mean number of 2.5 PIPAC ( \pm 1.5 ) (Fig. 1$) .64 .3 \%$ and $47.5 \%$ of patients had at least 2 or 3 PIPAC procedures, respectively. Further procedural details are clarified in Table 2.

In 48 patients, PIPAC was associated with a type of toxicity CTCAE 4.0 grade 1 to 5 with a total of 55 adverse events. However grade 1 toxicities represented almost a half of them. Grade 4 and 5 were extremely rare $(<1 \%$ each). Grade 3 toxicities were encountered in 14 cases (13.9\%) (Table 3). Abdominal pain was present in $22.8 \%$ of all patients. $8.9 \%$ of patients had grade 3 pain, and $3 \%$ required a change of the PIPAC drug. This data was not available for 1 patient. Surgical complications grade 3 and 4 were accounted for in 4 patients (two hematomas, two surgical site infections) whereas one respiratory grade 5 toxicity was recorded in a patient with a preexistent respiratory condition.

Follow-up after the first PIPAC had a median of 5 months (IQR: 5-11). 50 patients (49.5\%) noted improvement of symptoms under PIPAC treatment. Median overall survival (OS) was calculated from the initial diagnosis of the primary tumor (102 months \pm 46.8 ), from the diagnosis of the peritoneal metastatic disease (not reached) and from the first PIPAC (not reached), respectively. The Kaplan Meier curves are presented in Fig. 2.

There was no relationship in the binary logistic regression analysis between any of the specified factors and CTCAE complications grade 3 or higher. There was no relationship in the binary logistic regression analysis between any of the specified factors and the presence of severe abdominal pain (grade 2 or higher) except for age over 65 where p-value was as the threshold of significance $(\mathrm{p}=0.055)$. In linear regression, age over 65 was significantly related to abdominal pain.

Univariate analysis (ANOVA) did not identify any prognostic factors for early discontinuation of the planned treatment ( 3 cycles of PIPAC). Severe abdominal pain (grade 2 or higher) was one of the investigated factors and it was not retained. Univariate analysis (ANOVA) identified only a BMI lower than 20 as prognostic factor for impaired overall survival ( $p=0.038$ ). The association of male sex and age over 65 as well as the association of $\mathrm{BMI}<20$ and age $>65$ proved statistically significant $(\mathrm{p}<0.05)$. There were no statistically significant factors in the multivariate analysis. Cox regression analysis failed to identify significant differences in survival based on the aforementioned variables (gender, $\mathrm{BMI}<20$, primary tumor, presence of abdominal pain CTCAE grade 1 to 3 , presence of any grade 3 toxicities).

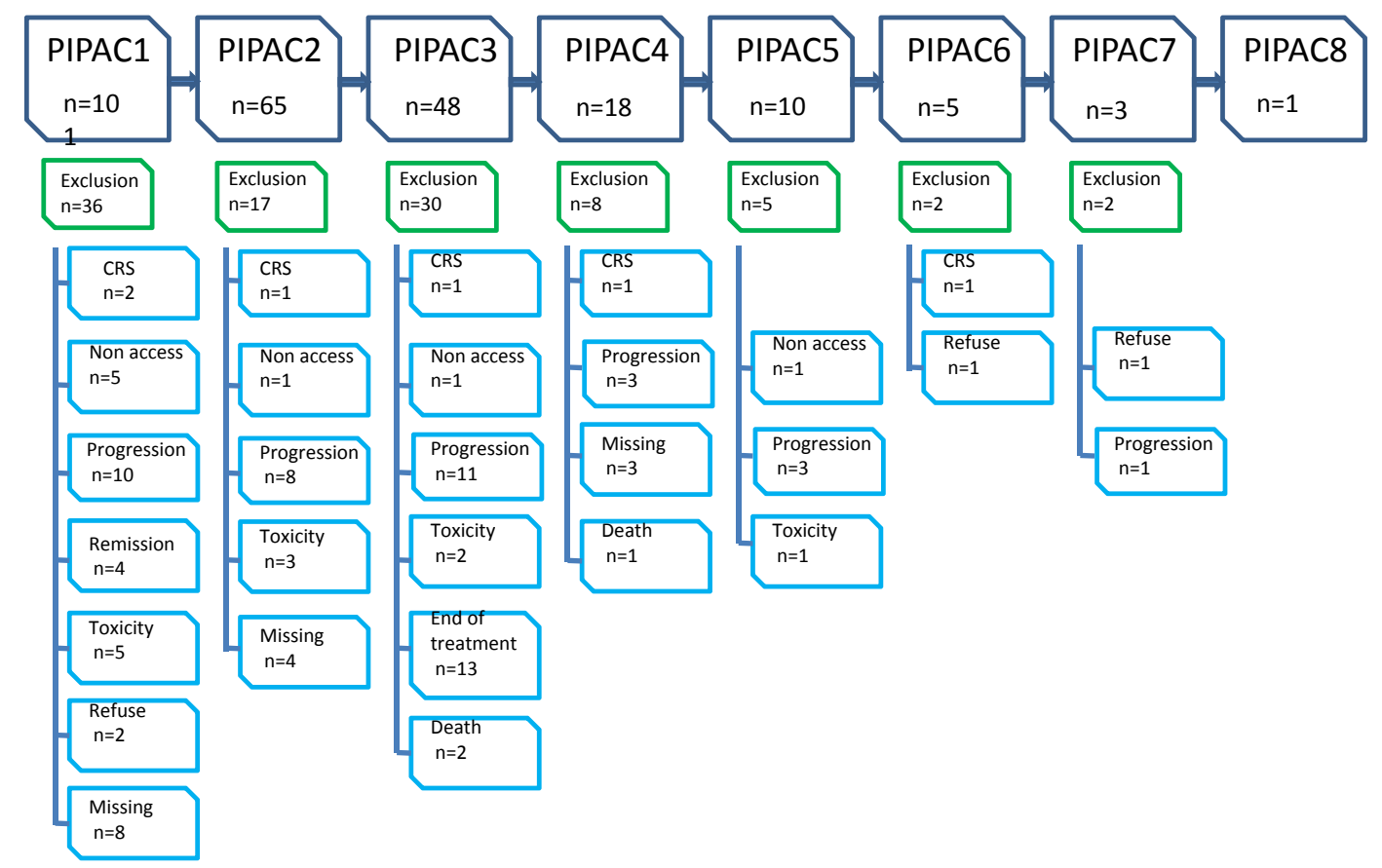

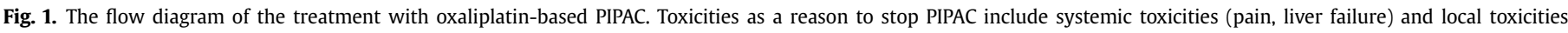
(hematoma, perforation). 
Table 2

Treatment-related characteristics of the patients in the present study (*the value is inferior to the total number of adverse events in Table 3 as several events can be described in the same patient).

\begin{tabular}{|c|c|c|c|}
\hline Variable & Subtype & Value & \\
\hline \multirow[t]{3}{*}{ Associated chemotherapy } & Yes & $47(46,5 \%)$ & \\
\hline & No & $6(5,9 \%)$ & \\
\hline & Missing & $48(47,6 \%)$ & \\
\hline \multirow[t]{6}{*}{$\begin{array}{l}\text { Median PCI for histological } \\
\text { subtypes }\end{array}$} & $\begin{array}{l}\text { Colorectal } \\
\text { cancer }\end{array}$ & $19(10,5-27,5)$ & $\mathrm{p}=\mathrm{NS}$ \\
\hline & Gastric cancer & $19(10-28)$ & \\
\hline & Ovarian cancer & $18[10-26]$ & \\
\hline & MPM & $\begin{array}{l}19[5,5-11,11 \\
-26]\end{array}$ & \\
\hline & PMP & $16,5[10-23]$ & \\
\hline & Other & $17,5(9,5-26)$ & \\
\hline \multirow[t]{8}{*}{ Median PCI per PIPAC cycle (IQR) } & PIPAC1 & $19(10-28)$ & \\
\hline & PIPAC2 & $19[14-26]$ & \\
\hline & PIPAC 3 & $20(15-27)$ & \\
\hline & PIPAC 4 & $14[8-22]$ & \\
\hline & PIPAC 5 & $20(14-30)$ & \\
\hline & PIPAC 6 & $12[10-19]$ & \\
\hline & PIPAC 7 & $17,5(-)$ & \\
\hline & PIPAC8 & $17(-)$ & \\
\hline \multirow[t]{6}{*}{ Mean number of PIPAC } & $\begin{array}{l}\text { Colorectal } \\
\text { cancer }\end{array}$ & $2,36 \pm 1,59$ & $\mathrm{p}=\mathrm{NS}$ \\
\hline & Gastric cancer & $2,60 \pm 0,83$ & \\
\hline & Ovarian cancer & $2,20 \pm 1,1$ & \\
\hline & MPM & $2,33 \pm 1,53$ & \\
\hline & PMP & $3 \pm 2,83$ & \\
\hline & Other & $3,2 \pm 2,04$ & \\
\hline \multicolumn{2}{|l|}{ Median length of stay (IQR) } & $3[2,3]$ days & \\
\hline \multicolumn{2}{|l|}{ Secondary non-access } & $8(7,9 \%)$ & \\
\hline \multicolumn{2}{|l|}{ Symptom response } & $50(49,5 \%)$ & \\
\hline \multicolumn{2}{|l|}{ CTCAE grade 3 or higher } & $16(15,9 \%)$ & \\
\hline \multicolumn{2}{|c|}{ Patients presenting with any grade toxicity* } & $37(36,6 \%)$ & \\
\hline \multicolumn{2}{|l|}{ Post-PIPAC cytoreductive surgery } & $6(5,9 \%)$ & \\
\hline
\end{tabular}

\section{Discussions}

In this multi-center study, PIPAC with oxaliplatin $92 \mathrm{mg} / \mathrm{m}^{2}$ appeared to be a safe and repeatable locoregional treatment for PC of various origins. High-grade adverse events occurred in only $15.9 \%$ of all patients, symptom response was accounted in half of the patients and encouraging survival curves call for further prospective evaluation.

Although oxaliplatin is one of the three validated drugs administered in $\operatorname{PIPAC}(9 ; 10 ; 16)$, it is still used with reluctance because of previous data showing CTCAE 4.0 grade 3 toxicities in $23 \%$ of patients(13). Furthermore, recent results from trials based on other intraperitoneal uses of oxaliplatin raised concerns with regards to the efficacy of this drug in the intraperitoneal setting(17). Unlike in hyperthermic intraperitoneal chemotherapy (HIPEC), oxaliplatin is administered repeatedly in PIPAC and under pressure conditions which enhance tissue entry(18) of a molecule with already satisfying pharmacokinetic properties (molecular weight 397.3 Da, area under the curve (AUC) peritoneum-plasma ratio 16) [19] used at five-times lower doses.

Toxicity studies on oxaliplatin-based PIPAC included reports of severe hypersensitivity(20) and peritoneal sclerosis(21). Severe hypersensitivity rates were $2.8 \%$ for oxaliplatin versus $0.6 \%$ for cisplatin-doxorubicin in a monocentric cohort of more than 300 PIPAC. In the colorectal PM cohort, CTCAE grade 3 events were observed in 4/17 patients (23\%), and no CTCAE 4 side effects were documented. Postoperative pain of all grades was reported in 11 cases (64.7\%) [13]. In comparison, toxicity rates of systemic administration of oxaliplatin are considerably higher with a $50 \%$ rate of neuropathy under treatment which persists in $0.7 \%$ of patients beyond 18 months after treatment(22). In addition, systemic oxaliplatin administration entails a $40 \%$ risk of transient thrombocytemia of any grade and of $12-19 \%$ hypersensitivity reactions(22). None of these effects has been observed after PIPAC-Ox with the current dose regimen.

PIPAC procedures combining cisplatin-doxorubicin were also associated with CTCAE 4.0 grade 3 or higher toxicities ranging from 16 to $36 \%$ in phase I, phase II and cohort studies $(2 ; 4 ; 23 ; 24)$. In the feasibility phase II study, there were 16\% CTCAE 4.0 grade 3 toxicities but only $4 \%$ abdominal pain grade $3(23)$. In the phase I study testing increasing doses of the cisplatin-doxorubicin association, only one grade 3 event (colon perforation, surgery-related) was described and it belonged to the first dose level(4). In a cohort of patients with gastric cancer treated with PIPAC C/D, grade 3-5 toxicities were encountered in $36 \%$ of the patients ( $25 \%$ grade 3 ). The present PIPAC-Ox results are similar to that of the cisplatindoxorubicin phase II study and superior to previous PIPAC C/D and PIPAC-Ox reports.

In the present study, $22.8 \%$ of all patients presented with abdominal pain but only $9 \%$ of them had grade 3 abdominal pain while only $3 \%$ needed a change in the PIPAC drug due to this toxicity. These figures are higher than those published for cisplatindoxorubicin but there is no proof of significant impact on the treatment in regression analysis. Practices of pain management after the PIPAC procedure vary among the investigating centers with some centers routinely offering opioids after PIPAC-Ox which can interfere with the results. The change of the PIPAC drug was performed when grade 3 abdominal pain was persistent after 1 week use of high level analgesia (opioids or derivates). Unfortunately the quality of life data was not available for all the patients in the study. Also inflammatory response was not tested as postoperative inflammatory response was similar in a previous cohort study comparing PIPAC C/D versus PIPAC-Ox(25).

Following regression, univariate and multivariate analysis, very few prognostic factors can be identified, probably due to the heterogeneity of the patients in this cohort. The selection criteria for patients undergoing PIPAC evolved since the initial experience of some of the centers that participated in this study therefore

Table 3

Frequency and grade of main toxicities encountered after PIPAC.

\begin{tabular}{|c|c|c|c|c|c|c|}
\hline Toxicity & Grade I & Grade II & Grade III & Grade IV & Grade V & Total \\
\hline Abdominal pain & 11 & 3 & 9 & - & - & 23 \\
\hline Hematologic & 0 & 2 & 0 & 0 & 0 & 2 \\
\hline Cardiac & 0 & 0 & 0 & 0 & 0 & 0 \\
\hline Gastrointestinal & 4 & 5 & 1 & 0 & 0 & 10 \\
\hline Respiratory & 0 & 0 & 0 & 0 & 1 & 1 \\
\hline Renal & 1 & 1 & 0 & 0 & 0 & 2 \\
\hline Hepatic & 4 & 0 & 1 & 0 & 0 & 5 \\
\hline Allergic & 2 & 0 & 1 & 1 & 0 & 4 \\
\hline Surgical (parietal abcess/hematoma) & 0 & 6 & 2 & 0 & 0 & 8 \\
\hline Total & 22 & 17 & 14 & 1 & 1 & 55 \\
\hline
\end{tabular}


Survival Function

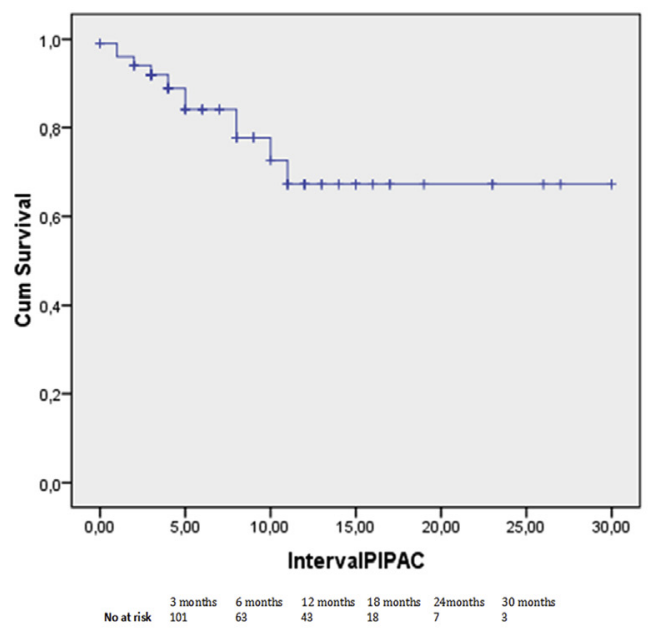

Survival Functions

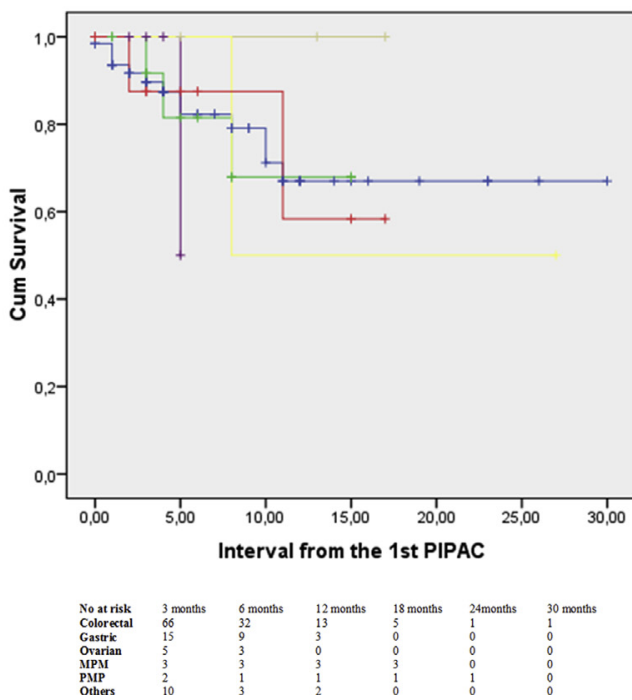

Primary cancer

$\rightarrow$ colorecta

$\rightarrow$ gastric

-
PMParian

$\Pi$ others

$+1,00$-censore

$+2,00-$ censored

3,00-censore $+4,00$-censored

Fig. 2. Kaplan Meier survival curves reported to the first PIPAC administration a) for the entire group; b) for the different histological types of PC.

surgical complications and the postoperative onset of bowel obstruction have diminished(2). On the other hand, the symptom improvement rate following PIPAC remains similar at $49.5 \%$.

The survival curves following oxaliplatin-based PIPAC are very encouraging with median survival not reached for the global group or for the colorectal subgroup. Overall survival was $62 \%$ at 30 months for the entire group as well as for the colorectal patients which compares favorably with OS rates for PM patients under systemic chemotherapy(26). The other groups based on the histology of the primary tumor were scarcely represented therefore results should not be extrapolated. However these encouraging survival data, although not definitive in the absence of randomized controlled trials, demonstrate once more that advances were made in patient selection and systemic treatment association when compared to earlier studies $(2 ; 13)$.

Main limitations of the present study are its retrospective study design, limited patient sample, heterogeneous indications, and absence of control group. However, treatment was highly standardized as shown in a recent study(16). Then, deliberate patient selection appears to be unlikely given the high PCI at first PIPAC, even for primary etiologies where peritoneal surgery is not a standard. Furthermore, PIPAC was utilized in larger terms in clinical practice only since 2015 and the present study provides the largest overview on PIPAC-Ox so far.

In summary, PIPAC with oxaliplatin $92 \mathrm{mg} / \mathrm{m}^{2}$ appears to be a feasible and safe treatment alternative for patients with advanced PC. It offers symptom improvement in half of the patients, and short-term survival data is favorable. Dose-escalation studies are underway, and ongoing and planned phase II and III comparative trials should help to define potential indications for PIPAC-Ox.

\section{Declaration of interest}

None.

\section{References}

[1] Solass W, Hetzel A, Nadiradze G, Sagynaliev E, Reymond MA. Description of a novel approach for intraperitoneal drug delivery and the related device. Surg Endosc 2012;26(7):1849-55.

[2] Alyami M, Gagniere J, Sgarbura O, Cabelguenne D, Villeneuve L, Pezet D, et al. Multicentric initial experience with the use of the pressurized intraperitoneal aerosol chemotherapy (PIPAC) in the management of unresectable peritoneal carcinomatosis. Eur J Surg Oncol 2017;43(11):2178-83.

[3] Solass W, Kerb R, Murdter T, Giger-Pabst U, Strumberg D, Tempfer C, et al Intraperitoneal chemotherapy of peritoneal carcinomatosis using pressurized aerosol as an alternative to liquid solution: first evidence for efficacy. Ann Surg Oncol 2014;21(2):553-9.

[4] Tempfer CB, Giger-Pabst U, Seebacher V, Petersen M, Dogan A, Rezniczek GA A phase I, single-arm, open-label, dose escalation study of intraperitoneal cisplatin and doxorubicin in patients with recurrent ovarian cancer and peritoneal carcinomatosis. Gynecol Oncol 2018;150(1):23-30.

[5] Elias D, Bonnay M, Puizillou JM, Antoun S, Demirdjian S, El OA, et al. Heated intra-operative intraperitoneal oxaliplatin after complete resection of peritoneal carcinomatosis: pharmacokinetics and tissue distribution. Ann Oncol 2002;13(2):267-72.

[6] Dumont F, Senellart H, Pein F, Campion L, Glehen O, Goere D, et al. Phase I/Il study of oxaliplatin dose escalation via a laparoscopic approach using pressurized aerosol intraperitoneal chemotherapy (PIPOX trial) for nonresectable peritoneal metastases of digestive cancers (stomach, small bowel and colorectal): rationale and design. Pleura and Peritoneum; 2018.

[7] Kim G, Tan HL, Chen E, Teo SC, Min Jang CJ, Ho J, et al. Study protocol: phase 1 dose escalating study of pressurized intra-peritoneal aerosol chemotherapy (PIPAC) with oxaliplatin in peritoneal metastasis. Pleura and Peritoneum; 2018.

[8] Kurtz F, Struller F, Horvath P, Solass W, Bosmuller H, Konigsrainer A, et al. Feasibility, safety, and efficacy of pressurized intraperitoneal aerosol chemotherapy (PIPAC) for peritoneal metastasis: a registry study. Gastroenterol Res Pract 2018:2018:2743985.

[9] Grass F, Vuagniaux A, Teixeira-Farinha H, Lehmann K, Demartines N, Hubner M. Systematic review of pressurized intraperitoneal aerosol chemotherapy for the treatment of advanced peritoneal carcinomatosis. Br J Surg 2017:104(6):669-78.

[10] Tempfer C, Giger-Pabst U, Hilal Z, Dogan A, Rezniczek GA. Pressurized intraperitoneal aerosol chemotherapy (PIPAC) for peritoneal carcinomatosis: systematic review of clinical and experimental evidence with special emphasis on ovarian cancer. Arch Gynecol Obstet 2018;298(2):243-57.

[11] Sgarbura O, Samalin E, Carrere S, Mazard T, de Forges H, Alline M, et al. Preoperative intraperitoneal oxaliplatin for unresectable peritoneal carcinomatosis of colorectal origin: a pilot study. Pleura and Peritoneum 2016;1(4): 209-15.

[12] Levine EA, Votanopoulos KI, Shen P, Russell G, Fenstermaker J, Mansfield P, et al. A multicenter randomized trial to evaluate hematologic toxicities after hyperthermic intraperitoneal chemotherapy with oxaliplatin or mitomycin in patients with appendiceal tumors. J Am Coll Surg 2018;226(4):434-43.

[13] Demtroder C, Solass W, Zieren J, Strumberg D, Giger-Pabst U, Reymond MA. Pressurized intraperitoneal aerosol chemotherapy with oxaliplatin in colorectal peritoneal metastasis. Colorectal Dis 2016;18(4):364-71.

[14] Giger-Pabst U, Tempfer CB. How to perform safe and technically optimized pressurized intraperitoneal aerosol chemotherapy (PIPAC): experience after a consecutive series of 1200 procedures. J Gastrointest Surg 2018;22(12): 2187-93.

[15] Hubner M, Grass F, Teixeira-Farinha H, Pache B, Mathevet P, Demartines N. Pressurized IntraPeritoneal aerosol chemotherapy - practical aspects. Eur J Surg Oncol 2017;43(6):1102-9. 
[16] Nowacki M, Alyami M, Villeneuve L, Mercier F, Hubner M, Willaert W, et al. Multicenter comprehensive methodological and technical analysis of 832 pressurized intraperitoneal aerosol chemotherapy (PIPAC) interventions performed in 349 patients for peritoneal carcinomatosis treatment: an in ternational survey study. Eur J Surg Oncol 2018;44(7):991-6.

[17] Ceelen W. HIPEC with oxaliplatin for colorectal peritoneal metastasis: the end of the road? Eur J Surg Oncol 2019 Mar;45(3):400-2.

[18] Solass W, Herbette A, Schwarz T, Hetzel A, Sun JS, Dutreix M, et al. Therapeutic approach of human peritoneal carcinomatosis with dbait in combination with capnoperitoneum: proof of concept. Surg Endosc 2012;26(3):847-52.

[19] Sugarbaker PH, Van der Speeten K. Surgical technology and pharmacology of hyperthermic perioperative chemotherapy. J Gastrointest Oncol 2016;7(1): 29-44.

[20] Siebert M, Alyami M, Mercier F, Gallice C, Villeneuve L, Berard F, et al. Severe hypersensitivity reactions to platinum compounds post-pressurized intraperitoneal aerosol chemotherapy (PIPAC): first literature report. Cancer Chemother Pharmacol 2018.

[21] Graversen M, Detlefsen S, Pfeiffer P, Lundell L, Mortensen MB. Severe peritoneal sclerosis after repeated pressurized intraperitoneal aerosol chemotherapy with oxaliplatin (PIPAC OX): report of two cases and literature survey.
Clin Exp Metastasis 2018;35(3):103-8.

[22] Hoff PM, Saad ED, Costa F, Coutinho AK, Caponero R, Prolla G, et al. Literature review and practical aspects on the management of oxaliplatin-associated toxicity. Clin Colorectal Cancer 2012;11(2):93-100.

[23] Tempfer CB, Winnekendonk G, Solass W, Horvat R, Giger-Pabst U, Zieren J, et al. Pressurized intraperitoneal aerosol chemotherapy in women with recurrent ovarian cancer: a phase 2 study. Gynecol Oncol 2015;137(2):223-8.

[24] Nadiradze G, Giger-Pabst U, Zieren J, Strumberg D, Solass W, Reymond MA. Pressurized intraperitoneal aerosol chemotherapy (PIPAC) with low-dose cisplatin and doxorubicin in gastric peritoneal metastasis. J Gastrointest Surg 2016;20(2):367-73.

[25] Teixeira FH, Grass F, Labgaa I, Pache B, Demartines N, Hubner M. Inflammatory response and toxicity after pressurized IntraPeritoneal aerosol chemotherapy. J Cancer 2018;9(1):13-20.

[26] Franko J, Shi Q Meyers JP, Maughan TS, Adams RA, Seymour MT, et al. Prognosis of patients with peritoneal metastatic colorectal cancer given systemic therapy: an analysis of individual patient data from prospective randomised trials from the analysis and research in cancers of the digestive system (ARCAD) database. Lancet Oncol 2016;17(12):1709-19. 